\title{
RAUMPLAN Y EL VALOR COMPOSITIVO DE LA GEOMETRÍA BASADA EN EL CUBO.
}

\section{RAUMPLAN AND THE COMPOSITIVE VALUE OF THE GEOMETRY BASED ON THE CUBE.}

\author{
Yannette Díaz Umaña ${ }^{1}$ \\ Mawency Vergel Ortega ${ }^{2}$ \\ Julio Alfredo Delgado Rojas ${ }^{3}$ \\ Universidad Francisco de Paula Santander.
}

$6 \begin{array}{lll}6 & 0 & 2\end{array}$

\section{RESUMEN:}

Este artículo surge desde la práctica docente con la asignatura de Historia de la arquitectura moderna, vinculada al proyecto investigativo que integra la matemática y la arquitectura en los procesos de enseñanza aprendizaje. De esta forma se estudia la rigurosidad geométrica de la estrategia proyectual de Adolf Loos apoyada en el sistema Raumplan, aplicado a unidades espaciales autónomas configuradas a partir del cubo. Se identifica una maniobra

$1 \quad$ Magister en Gestión Urbana, Arquitecta, docente y directora del Departamento de Arquitectura, diseño y Urbanismo. Filiación: Universidad Francisco de Paula Santander. Correo electrónico: yannettedu@ufps.edu.co Orcid: https://orcid.org/0000-0003-4582-1593

2 Doctora en Educación. Postdoctora en Imaginarios y representaciones sociales. Docente y Directora del Departamento de Matemáticas y Estadística. Filiación: Universidad Francisco de Paula Santander. Correo electrónico: mawency@ufps.edu.co. Orcid: https://orcid.org/0000-00018285-2968

3 Magister en Educación Matemática, Arquitecto, Docente. Filiación: Universidad Francisco de Paula Santander. Correo electrónico: julioalfredo@ufps.edu.co Orcid: https://orcid.org/0000-0001-6944-832X de trabajo escultórico ordenado y lógico, cuya representación $3 \mathrm{~d}$ deja ver el pensamiento tectónico de Loos, incapaz de comprenderse totalmente desde un plano o fotografía. A través del análisis de la casa Steiner con una metodología descriptiva con paradigma cualitativo y cuantitativo, se analiza la destreza de disponer, sustraer y combinar las piezas en medio de un juego binario donde la fachada principal versus fachada posterior, interior versus exterior, se perciben como elementos antagónicos de un mismo volumen compacto cubico, demostrando la comprensión de la calidad espacial vista en los modos de habitar.

\section{PALABRAS CLAVE}

Geometría, cubo, arquitectura, modernidad, Raumplan.

\section{SUMMARY}

This article arises from the teaching practice with 
the subject of History of modern architecture, linked to the research project that integrates mathematics and architecture in the teachinglearning processes. In this way, the geometric rigor of Adolf Loos' project strategy based on the Raumplan system, applied to autonomous spatial units configured from the cube, is studied. An orderly and logical sculptural work maneuver is identified, whose $3 \mathrm{~d}$ representation reveals Loos's tectonic thought, unable to be fully understood from a plane or photograph. Through the analysis of the Steiner house with a descriptive methodology with a qualitative and quantitative paradigm, the skill of arranging, subtracting and combining the pieces is analyzed in the middle of a binary game where the main facade versus rear facade, interior versus exterior, are perceived as antagonistic elements of the same compact cubic volume, demonstrating the understanding of the spatial quality seen in the ways of living.

\section{KEYWORDS}

Geometry, cube, architecture, modernity, Raumplan.

\section{INTRODUCCIÓN}

A lo largo de la historia de la humanidad se ha podido apreciar el valor que ha tenido la geometría en el diseño de proyectos, llegando a ser clave para la composición arquitectónica. De esta manera se puede determinar a la geometría como una herramienta de representación e interpretación del mundo, y se identifica en una línea pluridisciplinaria de las matemáticas (Camargo y Acosta 2012). Estos autores comentan la utilidad: "En su dimensión biológica, se relaciona con capacidades humanas como el sentido espacial, la percepción y la visualización. En su dimensión física, indaga por propiedades espaciales de los objetos físicos y de sus representaciones, modelando el espacio circundante." (pág. 4).
En esta apreciación se pueden identificar el uso estratégico de la geometría en el movimiento moderno y contemporáneo, en especial las propuestas de Adolf Loos, Le Corbusier, Giuseppe Terragni, entre otros, ya que utilizan la geometría para definir los límites del espacio y asentar una arquitectura racionalmente pensada en el orden y la economía (Díaz, Delgado, \& Vergel 2021).

La arquitectura de Loos pone especial énfasis en el diseño de los elementos que regulan la composición, porque además de proponer un diseño diferente a la tradicional arquitectura del momento, confronta radicalmente el uso del ornamento. Es así, que sus escritos y obras arquitectónicas provocaron un revuelo generalizado, causado por una propuesta ética, estética y económica, planteada en 1908 desde "Ornamento y delito" (Loos 1908) y publicado en varios idiomas. De modo que sus planteamientos se desarrollaron, de cierta forma en contraste con los estilos de la Secesión Vienesa y en general el modernismo europeo. El propio Le Corbusier consideró la publicación de Ornamento y Delito de Loos como "una limpieza homérica" de la arquitectura, que revela la magnitud de su impacto en la ideología modernista (Rawn 2014).

\section{APROXIMACIONES AL PLAN DE VOLÚMENES DE LOOS Y LA RACIONALIDAD CONSTRUCTIVA DESDE LA GEOMETRÍA.}

La estrategia de Loos persigue la rigurosidad geométrica de las formas concebidas en un sólido compacto desarrollado a partir de la planta preferiblemente cuadrada, que al elevarse en altura conforma un cubo. Bajo el sistema Raumplan o plan de volúmenes se da un orden jerárquico -por cuanto las dimensiones de cada espacio-, continuo -dado por las relaciones espaciales-, y complejo -conexo a sus usos-. De ahí, este plan, lo hace diferente de los arquitectos del momento: "Es distinto el 
caso de Loos, que intento la conformación de una arquitectura autónoma y pura" (De Fusco 1992, p.171).

De esta manera Loos no abandona el propósito de la elegancia con el uso magistral de los acabados, pero se inclina por la interpretación del modo de vida de las personas que habitan los espacios, desde la comprensión de los intereses y deseos de los usuarios, resueltos en el uso racional de los espacios. Es evidente que a través de un sólido compacto rescata de forma abstracta el valor de las tradiciones y los tiempos pasados, pero no busca en ellos una referencia estilística directa. Desarrolla un proceso mental donde abstrae toda esta información decantándola en la simplicidad de las formas cubicas. En efecto, este arquitecto se reveló contra el estilo modernista imperante, para proponer un modelo de arquitectura rigurosa y funcional, en la que se prescinde de toda ornamentación.

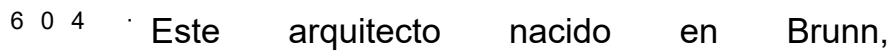
Checoslovaquia en 1870, fue quien abrió camino a una experiencia de diseño basada en el racionalismo y la geometría en Europa a finales del siglo XIX y principios del XX. Se caracterizaba por su discurso intelectual que prefiguró las bases del movimiento moderno, aportando posteriormente conceptos aplicados también en la contemporaneidad. Esto se explica analizando obras como la villa Savoye, casa Fascio en la modernidad y Caixa Forum Madrid, Mediateca Sendai en la contemporaneidad, cuyo desarrollo formal se vincula a la capacidad para apilar niveles en un sólido poliédrico. De allí se puede encontrar en la contemporaneidad, planteamientos que enaltecen la robustez, la gravedad del objeto construido y la capacidad expresiva de los materiales (Caixa Forum Madrid y Dominius Winery). Para otros, el antecedente Loossiano es tomado como la concepción basada en un cubo donde cada uno de los espacios con diferentes magnitudes comparten un techo, gracias a la utilización de las técnicas del hormigón armado y el acero (Díaz, Díaz y Centurión 2021). Aun cuando en esta última variante las envolventes sean ingrávidas y transparentes (Mediateca Sendai, museo del vidrio), las resoluciones internas de los espacios, realzan la racionalidad constructiva compacta desde la geometría (Díaz, Díaz y Centurión 2021).

Volviendo al caso de Loos, como antecedente creativo, la construcción de forjados continuos, combinando la simpleza de las formas tradicionales con materiales lujosos perfiladas por las geometrías más simples, lleva implícita una consecuencia añadida: que la consistencia o lógica visual, prevalece sobre la constructiva (Díaz, Meri de la Maza y Serra, 2017 pág. 24). En efecto la resolución espacial se da por la experimentación en el sólido, mas no, desde su sistema estructural, como generador y ordenador de los ambientes. Dicho de otra forma, el objetivo de Loos es alcanzar el orden perceptivoexpresivo por encima de la capacidad estructural, de ahí que defienda la racionalidad basada en la búsqueda geométrica y la expresividad en los materiales del revestimiento, pero no en los elementos estructurales (Peterson [1980] 2018 citado en (Meri de la Maza y Serra, 2017 pág. 24).

Fueron varias las obras de Adolf Loos que mostraron total coherencia con la intención de hallar pureza, economía y rigurosidad geométrica, asociadas al sistema Raumplan o plan de volúmenes. La casa Steiner (1910) reafirma la habilidad proyectiva de Loos en este aspecto conciliando la normatividad del sector y los requerimientos del cliente. Moneo afirma: "Quien quiera que construya sabe que encerrar el más amplio volumen en la menor de las superficies es siempre deseable: hay una recompensa formal implícita en toda arquitectura respetuosa con la economía intrínseca "(Moneo 2010, pág. 471). Con esta idea se comprende 
que "La economía conduce a ocupar -y, consecuentemente, programar- la sección de la manera más compacta posible y, por tanto, ayuda a configurar la sección libre vinculada al Raumplan de Loos." (Toral 2018, pág. 81).

\section{DISCUSIÓN}

De esta forma, la casa Steiner se alza en un cubo casi perfecto de $14.5 \mathrm{~m} \times 13.5 \mathrm{~m}$ $x 13 m$ cuyo volumen está constituido por yuxtaposición de espacios interiores de diferentes alturas conservando los limites del poliedro. Exteriormente, el alzado principal, "decididamente feo" como lo indica De Fusco (1992), ubicado sobre la calle, desvirtúa la verdadera magnitud de la altura, insertándose en el terreno y ocultando gran parte de los niveles: sótano, primer piso y ático, estos dos últimos ocultos tras la cubierta curvada en chapa. A su vez, la fachada posterior sobre el jardín, visualmente más alta, desnuda y simplemente clasista, arregla una dicotomía mediada por la imparcialidad de los muros laterales de la casa. Hernández (1990) expresa con ello distintos juegos binarios: fachada anterior versus posterior, interior versus exterior, lo que significa configurar los planos del solido de manera distinta y sorpresiva para el espectador. Este juego se extiende del mismo modo al manejo de los materiales cálidos, domésticos y finos al interior y fríos, austeros y económicos al exterior, por consiguiente, se proyectan fuertes contrastes que se alinean con la reflexión de Loos al promover que la envolvente del solido no debe decir nada de su interior. (ver figura 1)

Figura1. Casa Steiner volumetría general, conceptualización cubica compositiva.
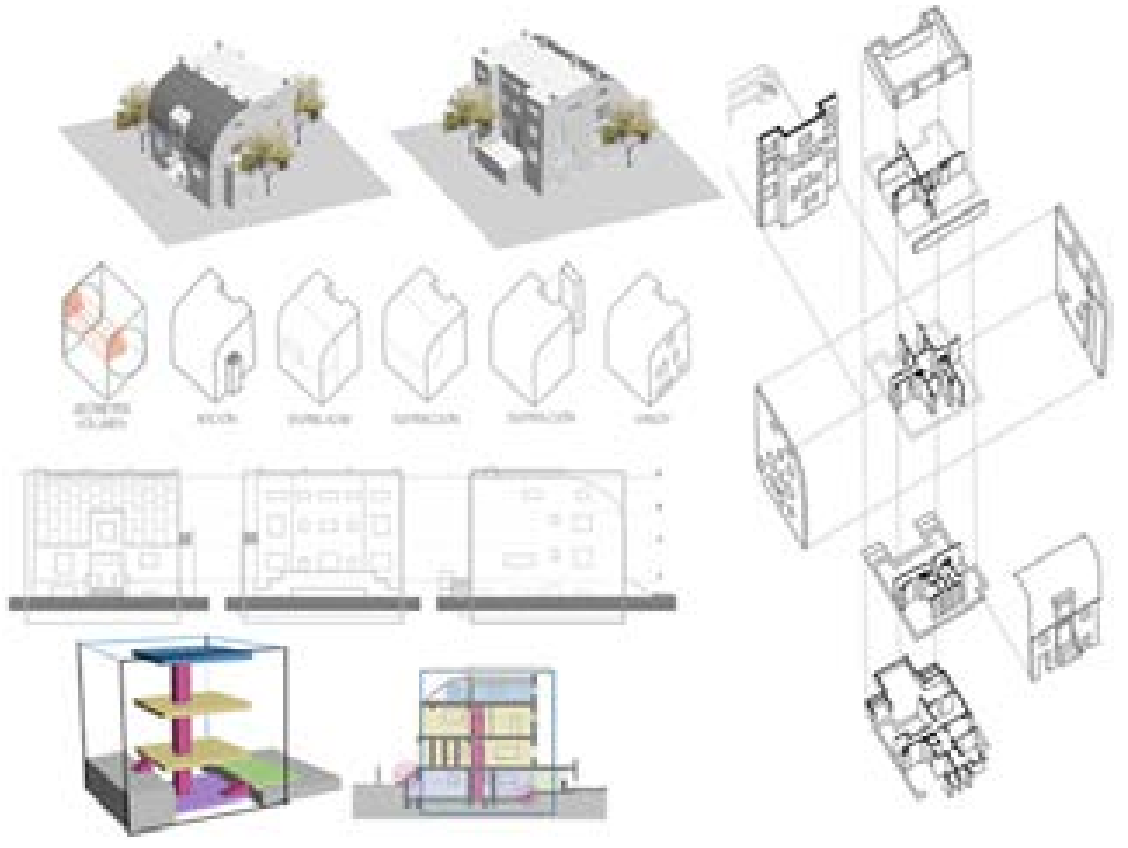

Fuente: elaborado por Maria Valentina Gonzalez.

Esta separación radical entre el interior y el exterior que se observa en la casa Steiner, protege la intimidad de la vivienda, así su carácter doméstico e íntimo solo concierne al usuario, en este caso, sus dueños la pintora
Lilly Steiner y su esposo Hugo Steiner. En contraste, al exterior, una piel económica en revoque blanco, limpia -en lo pertinente al ornamento- $y$ ahuecada en ciertos lugares estratégicos, dispuestos solo a los intereses de 
quien observa desde el interior a través de los pequeños vacíos- ventanas- en las caras del cubo. Por otra parte, el eje de la distribución en torno al cual giran las plantas, es el núcleo principal, ubicado con centralidad geométrica en el interior del cubo, también definido como el gradiente vertical que determina la continuidad perceptiva de los niveles y también conductor de las instalaciones. Este elemento vertical media las diferentes secciones en altura entre placas que corresponden a $4.0 \mathrm{~m}$ en el sótano, $3.0 \mathrm{~m}$ en la planta baja, $2.85 \mathrm{~m}$ en el primer piso y $2,10 \mathrm{~m}$ en el ático,

Díaz, Meri de la Maza y Serra (2017) describen en las obras de Loos, el trabajo de un escultor donde se ve un concepto tridimensional, "que quizá solo quien lo ha concebido puede entender completamente" (pág. 24). Es una estrategia proyectual donde la variedad de alternativas que aporta la geometría facilita una composición ordenada y lógica, en el que se consiguen unas formas basadas a partir del uso de la cuadrícula en $3 \mathrm{~d}$ ortogonal. Por su parte (Díaz, Delgado \& Vergel 2021) se refieren a este principio de composición, como la idea asociada a la de trama reguladora y ordenadora. (Díaz, Delgado \& Vergel 2021)

Otras de las interpretaciones frente al trabajo de este arquitecto es un juego de ajedrez en tres dimensiones, dejando en libertad los movimientos de las piezas, pero siempre en el interior de un volumen limitado denominado "cubo" (Hernández 1990). Al respecto Díaz, Meri de la Maza y Serra (2017) afirman que la representación del pensamiento tectónico de Loos no puede ser concebida desde un plano o fotografía ya que no pueden figurar fielmente los contenidos, por o tanto, la arquitectura de Loos implica un disponer y combinar las piezas entre ellas de forma tridimensional con relación al habitar.

Por eso, la concepción del Raumplan como pensamiento tectónico, va más allá de una propuesta formal o lingüística, pues la habilidad cognitiva vista en disponer, sustraer y combinar las piezas dentro del poliedro demuestra la comprensión de la calidad espacial vista en los modos de habitar. Esta operación de deconstrucción del cubo por medio de dividirlo o cortarlo para observar qué tiene por dentro, consiente el trabajo modular tridimensional (Restrepo, Martinez y Quiroga 2013), teniendo como su mayor expresión de simplicidad fundamentalmente por la direccionalidad de sus aristas que corresponden al sistema de coordenadas de mayor difusión (cartesiano ortogonal), y la facilidad de ocupar o rellenar modularmente el espacio tridimensional, sin dejar vacíos o superponerse (Serrentino, y Molina 2002 y Carvajal 2011).

De esta forma, Loos descubre la variedad de posibilidades que tiene un volumen finito, permitiendo una composición de vacíos y llenos, con variantes infinitas como se puede constatar en sus posteriores obras: la casas Tristan Tzara, Müller, Moller entre otras. De igual manera este juego geométrico fue lo suficientemente perceptivo para influir en la arquitectura contemporánea y en sus medios de graficación, ampliando la experimentación con los sistemas de diagramación digital $3 d$ que facilitan una mayor experimentación de este concepto modular. Así mismo el desarrollo de estrategias de enseñanza-aprendizaje de la forma arquitectónica con herramientas matemáticas y geométricas.

\section{CONCLUSIONES}

La experiencia constructiva del arquitecto Adolf Loos abre un camino sin precedentes al proponer una estrategia de diseño que regula la composición desde un concepto diferente a la tradicional arquitectura del momento, confrontando radicalmente el uso del ornamento. Con la intención de hallar pureza y economía persigue la rigurosidad geométrica de las formas concebidas en un sólido compacto desarrollado 
a partir de la planta preferiblemente cuadrada, que al elevarse en altura conforma un cubo. Las relaciones espaciales se rigen bajo el sistema Raumplan o plan de volúmenes dando un orden jerárquico, continuo y complejo como lo enseña creativamente en la Casa Steiner de 1910.

El pensamiento tectónico de Loos, aplica una estrategia compositiva de disponer, sustraer y combinar las piezas dentro del poliedro argumentado en la comprensión de los modos de habitar. Bajo este parámetro la casa Steiner que se alza como una figura sólida con criterios espaciales del Raumplan, en un cubo casi perfecto de $14.5 \mathrm{~m} \times 13.5 \mathrm{~m} \times 13 \mathrm{~m}$ cuyo volumen está constituido por yuxtaposición de espacios interiores de diferentes alturas, prefigura las bases del movimiento moderno. Permite abordar las estrategias de enseñanzaaprendizaje de la forma arquitectónica con herramientas matemáticas y geométricas, ampliando la experimentación con los sistemas de graficación digital $3 \mathrm{~d}$ desde racionalidad constructiva y la geometría.

\section{REFERENCIAS BIBLIOGRÁFICAS}

Camargo, Leonor y Acosta, Martín. (2012). La geometría, su enseñanza y su aprendizaje. Tecné, Episteme y Didaxis: TED, (32), 4-8. Obtenido el 3 de septiembre de 2021 de http://www.scielo.org.co/scielo. php?script $=$ sci arttext $\&$ pid $=$ S0121$38142012000200001 \&$ Ing=en\&tIng=es.

Carvajal, Javier. (2011). Secciones Modulares del Cubo. Madrid: Ed Jacar \& Dacar

De Fusco, Renato. (1992). "Historia de la arquitectura contemporánea”. Editorial celeste.

Díaz, Alfonso; Meri de la Maza, Ricardo y Serra, Bartolomé. (2017). La construcción del Raumplan. Actas I Congresolberoamericano redfundamentos.

http://ojs.redfundamentos.com/index.php/actas/ article/view/218

Díaz, Yannette, Díaz, Carmen y Centurión, Ramón (2021). 35 proyectos de arquitectura contemporánea. Materia, forma \& Contexto. Editorial Ecoe, Bogotá.

Díaz-Umaña, Y., Delgado-Rojas, J. A.., \& Vergel-Ortega, M. (2021). La geometría basada en la malla como mecanismo compositivo de Villa Savoye. Eco Matemático, 12(1). https://doi. org/10.22463/17948231.3070

Díaz Umaña, Y., Delgado Rojas, J. A.., \& Vergel Ortega, M. (2021). Estructura como envolvente en la iglesia nuestra señora del Carmen - Cúcuta. Revista Boletín Redipe, 10(8), 303-311. https://doi. org/10.36260/rbr.v10i8.1406

Hernández, Juan (1990). La casa de un solo muro. Editorial NEREA, S. A. Madrid

Moneo, Rafael. 2010. Apuntessobre21obras. Barcelona: Gustavo Gili.

Peterson, Steven Kent. [1980] 2018. "SpaceandAnti-Space". Actualizado en http://petersonlittenberg.com/ Architecture-UrbanDesign/Space_AntiSpace TOC.html

Rawn, Evan. (2014) "Adolf Loos, en perspectiva" [Spotlight: Adolf Loos] 10 dic 2014. ArchDaily Colombia. (Trad. Duque, Karina) https://www. archdaily.co/co/758865/adolf-loos-enperspectiva> ISSN 0719-8914 
Restrepo, Miryam: Martinez Yaquieline y Quiroga Maria (2013). Geometría de Patrones, una forma de llegar al vestido. 3 encuentro de Experiencias Investigativas del Vestiry la Moda. $\mathrm{Pa}$ (3342). https://d1wqtxts1xzle7.cloudfront. net/33200258/LIBRO._Experiencias Investigativas del vestir y la moda 3.pdf? $1394630748=$ \&responsecontent-disposition=inline $\% 3 \mathrm{~B}+$ filenam e\%3DMEMORIAS EXPERIENCIAS INVESTIGATIVAS DEL.pdf\&Expires=1 $\underline{630707517 \& \text { Signature }=}$

Serrentino, Roberto y Molina, Hernán (2002). Arquitectura modular basada en la teoría de policubos. Morfología y Matemáticas. pág. 264-267 http://papers.cumincad. org/data/works/att/2ed6.content.pdf

Toral Guinea, M. (2018). La función en los sistemas compactos de orden relacional. Cualificación espacial para una cierta flexibilidad. HipoTesis Serie Numerada, 6, 75-88. http://hipo-tesis. eu/serienumerada/index.php/ojs/article/ view/hipo6toral 